\title{
The Dynamics of Backfilling: Solving the Mystery of Why Increased Inaccuracy May Help
}

\author{
Dan Tsafrir* Dror G. Feitelson \\ School of Computer Science and Engineering \\ The Hebrew University, 91904 Jerusalem, Israel
}

\begin{abstract}
Parallel job scheduling with backfilling requires users to provide runtime estimates, used by the scheduler to better pack the jobs. Studies of the impact of such estimates on performance have modeled them using a "badness factor" $f \geq 0$ in an attempt to capture their inaccuracy (given a runtime $r$, the estimate is uniformly distributed in $[r,(f+1) \cdot r])$. Surprisingly, inaccurate estimates $(f>0)$ yielded better performance than accurate ones $(f=0)$. We explain this by a "heel and toe" dynamics that, with $f>0$, cause backfilling to approximate shortest-job first scheduling. We further find the effect of systematically increasing $f$ is V-shaped: average wait time and slowdown initially drop, only to rise again later on. This happens because higher $f s$ lead to increased randomness (more long jobs appear as short and vice versa) and to backfilling of longer jobs, overshadowing the "heel and toe" dynamics and limiting the preference for short jobs. Finally, we show that the badness factor fails to capture the badness of real estimates, because these are modal and bounded by a maximal value. Therefore the $f$ model is actually inappropriate for studying the effect of the inaccuracy of estimates.
\end{abstract}

\section{INTRODUCTION}

The workload on a parallel supercomputer consists of a sequence of jobs submitted for execution. These jobs are characterized by their arrival time, size (number of processors they need), runtime, and a runtime estimate provided by the user to aid the scheduler in planing ahead. Such estimates are known to be inaccurate, a fact that prompted many researchers to check their actual effect on the scheduling. Much of this research used the " $f$-model", in which estimates are assumed to be some multiple of the real runtime, and led to some surprising conclusions, such as the claim that inaccurate estimates result in improved performance. We explain this behavior, and show that this model is actually inappropriate for such research, as it does not reflect salient features of user estimates in real workloads. But we also show that a relatively minor modification improves the model and enables realistic evaluations.

Backfilling: The EASY backfilling algorithm [13] is currently the most commonly used method for parallel job scheduling, supported by all major production schedulers, including IBM's LoadLeveler, Cluster Resources' Moab and Maui, Platform's LSF, Altair's OpenPBS and PBS-Pro, and Sun's GridEngine [4]. Upon submittal, users specify the number of processors required by their jobs, and these are placed in a first-come first-serve (FCFS) queue. When enough free processors become available to fill the needs of the first queued job, it is allocated exclusive use of these processors and runs to completion. The

${ }^{*}$ Current affiliation: IBM T. J. Watson Research Center.
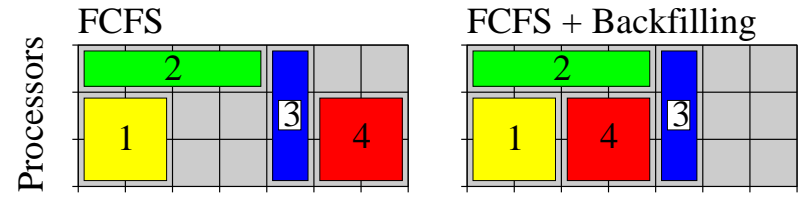

Future Time

Fig. 1. EASY backfilling reduces fragmentation, e.g. by running job 4 that is short enough to terminate before the reservation for job 3 .

problem with this approach is that many processors may be left idle as they accumulate. To solve this problem, the scheduler makes a reservation for the first queued job, for the earliest future time at which enough processors are expected to be available. The scheduler then continues to scan the queue for smaller jobs (requiring less processors) that can be started immediately without interfering with the reservation. The action of selecting such jobs for execution before their time is called backfilling. This is illustrated in Fig. 1.

User Runtime Estimates: Note that backfilling requires the scheduler to know in advance how long each job will run: to compute the reservation for the longest-waiting job (need to know the runtimes of job 1 and job 2 to determine when their processors will be freed), and to know if smaller jobs are short enough to be backfilled (need to make sure job 4 will terminate before the reservation of job 3). Therefore, EASY and other schedulers require users to provide a runtime estimate for all submitted jobs [13]. Jobs that exceed their estimates are killed, so as not to violate subsequent reservations. The assumption was that users would be motivated to provide accurate estimates, because (1) jobs would have a better chance to backfill if their estimates are tight, but (2) would be killed if they are too short. Nevertheless, user estimates are highly inaccurate [6], [12], [23].

Modeling Inaccuracy: To study the sensitivity of backfilling to poor estimates, Feitelson and Mu'alem Weil proposed the " $f$ model" [6]. Given a job $J$ with runtime $r$, the model postulates that its estimate is chosen at random from a uniform distribution in the range $[r,(f+1) \cdot r]$, where $f \geq 0$ is a predetermined constant ( $f$ is non-negative because jobs are killed if they try to run beyond their estimate). They termed $f$ the "badness factor" because estimates become increasingly inaccurate as $f$ grows, with $f=0$ indicating completely accurate estimates. The $f$-model has been used when simulating workloads that lacked estimates data [26], [8], but much more importantly, the 


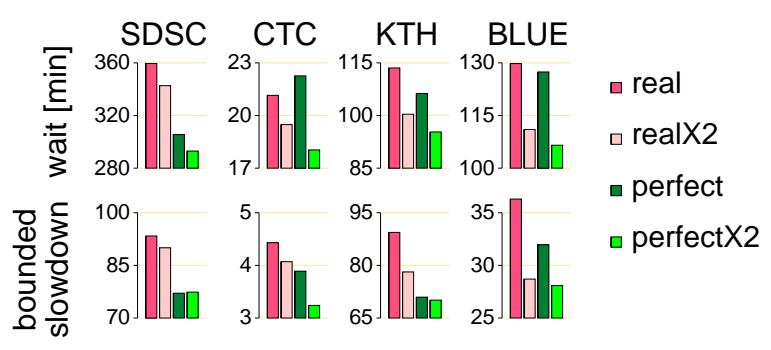

Fig. 2. Average performance usually improves if replacing user estimates ("real") by actual runtimes ("perfect"; $f=0$ ). But in both cases, making estimates less accurate (by doubling) tends to help.

model and its variants have been extensively used to study the impact of inaccurate estimates on backfilling algorithms [20], [14], [18], [27], [16], [9]. One simple variant of interest is the "deterministic $f$-model", in which there is no randomness and estimates are a direct multiple of the runtime and the badness factor plus one: $(f+1) \cdot r \quad$ [28], [1], [3].

The Inaccuracy Mystery: A very surprising result repeatedly reported by most of the aforementioned papers was that, in terms of performance, inaccurate estimates are usually preferable over accurate ones. This is illustrated in Fig. 2 that shows average wait time and slowdown of jobs when simulating the run of four different workloads with various estimates. Evidently, performance improves when deliberately making estimates less accurate by doubling them. This is true both when doubling perfectly accurate estimates and when doubling the original inaccurate user estimates [28], [14].

While there's wide agreement that making estimates less accurate by multiplying them by some factor is usually beneficial, the effect of the chosen $f$ is less obvious. This is illustrated in Fig. 3. Faced with (usually a small subset of) such results, researchers claimed that the improvement in performance is largely "insensitive" to $f$ [28], [26], [3], [9]. Further, England et al. suggested a new "robustness" metric for the evaluation of computer systems, and claimed (in a case-study demonstrating the usefulness of their metric) that [3]

"Our results support those of a previous work and also
indicate that backfilling is robust to inaccurate run time es-
timates in general. It seems that, with respect to backfilling,
what the scheduler doesn't know won't hurt it."

The Failure to Explain the Mystery: The fact nonzero badness $(f>0)$ usually improves performance was unanimously explained by what we call the "holes argument" [6], [28], [14], [1], [18], as articulated by Chiang et al. [1]:

\footnotetext{
"We note that for large $f$ (or when multiplying [real] estimates by two), jobs with long runtimes can have very large runtime overestimation, which leaves larger 'holes' for backfilling shorter jobs. As a result, average slowdown and wait may be lower"
}

At the same time, the observed "insensitivity" of performance to the exact badness value for $f>0$, was explained by what we call the "balance argument" [28], [26], [27], [9], as articulated by Zhang et al. [26]:

"We can understand why backfilling is not that sensitive
to the estimated execution time by the following reasoning.
On average, overestimation impacts both the jobs that
are running and the jobs that are waiting. The scheduler
computes a later finish time for the running jobs, creating
larger holes in the schedule. The larger holes can then be
used to accommodate waiting jobs that have overestimated
execution times. The probability of finding a backfilling can-
didate effectively does not change with the overestimation."

For example, doubling the lengths of all the jobs in Fig. 1 only means the $\mathrm{X}$-axis is scaled by a factor of two, but doesn't change anything regarding the backfilling decision: indeed, after doubling, job 4 looks twice as long in the eyes of the scheduler, but the same applies to the 2-time-units-hole opened by job 2 , so job 4 can still backfill.

While both arguments seemingly make sense, one obvious problem with them is that they are contradictory: If the balanceargument is correct, then there is no benefit in opening those "larger holes" as suggested by the holes-argument, because backfilling candidates would become proportionally larger and cancel the effect. On the other hand, the "holes argument" implies a performance improvement that is proportional to $f$, in contrast to the balance-argument rationale. Regardless of the contradiction, both arguments fail to explain the results shown in Fig. 3, for example the noisiness of BLUE (performance is actually quite sensitive to $f$ ), or the opposite trends observed in SDSC/wait vs. CTC/wait (CTC/wait supports the holesargument while SDSC/wait contradicts it; both contradict the balance-argument).

Failure to Capture Users" "Badness": The role of a model is, among other things, to truthfully reflect reality. In this respect, according to Fig. 3, the popular $f$-model fails: It yields unrealistically improved performance results that are consistently better than those obtained when real user estimates are utilized (the only exception is very small $f$ values in CTC/wait). Surprisingly, this issue has usually been brushed aside: In contrast to the other key parameters in parallel workloads (runtimes, interarrival times, number of processors) that received a lot of attention in terms of realistic modeling, the dominant estimate model has been the $f$-model or simply using actual runtimes instead of estimates $(f=0)$ [11], [19], [25], [5], [17]. We conjecture that this can be attributed, to some extent, to the perception that estimates are unimportant because "inaccuracy improves performance" and "what the scheduler doesn't know won't hurt it".

Preview: In this paper we try to solve all the questions raised above: Why are result so sensitive to $f$, as seen in Fig. 3? Why does multiplying the estimate by a factor usually help? 

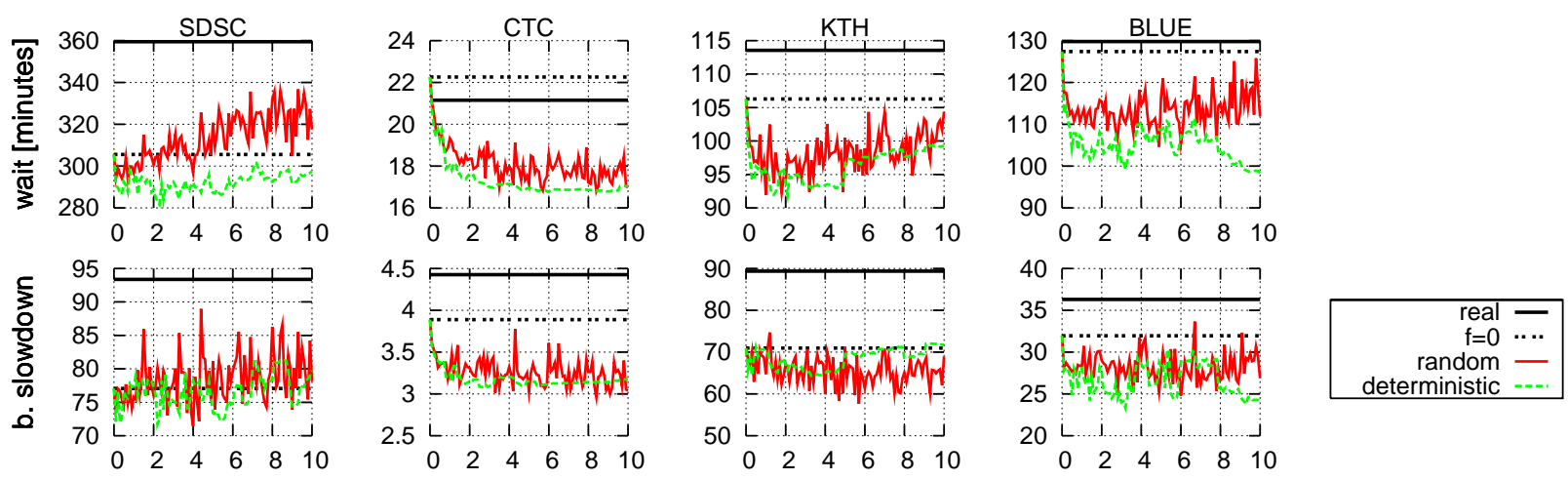

f (badness factor)

Fig. 3. Performance as a function of $f$ for the random and the deterministic $f$-model, with a resolution of 0.1 (that is, $f=0, \frac{1}{10}, \frac{2}{10}, \frac{3}{10}, \ldots, 10$ ). Except for SDSC, most results associated with positive $f$ values are better (smaller) than the performance associated with $f=0$ ("real" corresponds to real user estimates).

\begin{tabular}{|l|r|r|c|c|}
\hline \multicolumn{1}{|c|}{ Log file } & CPUs & \multicolumn{1}{c|}{ Jobs } & Duration & Util \\
\hline CTC-SP2-1996-1.1-cln & 512 & 77,222 & $6 / 96-5 / 97$ & $56 \%$ \\
KTH-SP2-1996-1 & 100 & 28,490 & $9 / 96-8 / 97$ & $69 \%$ \\
SDSC-SP2-1998-2.1-cln & 128 & 59,725 & $4 / 98-4 / 00$ & $84 \%$ \\
SDSC-BLUE-2000-2.1-cln & 1,152 & 243,314 & $4 / 00-6 / 03$ & $76 \%$ \\
\hline
\end{tabular}

The trace files used to drive the simulations.

Can we make the model more realistic? To do so, we perform a detailed study of what really happens when $f$ grows, both in terms of performance (Section III) and in terms of backfilling activity (Section IV). This leads to the heel-and-toe dynamics, which explain the improved performance as resulting from a shift in system behavior towards SJF scheduling (Section V). We then show why this breaks down with higher $f$ values (Section VI). The conclusion is that the $f$-model is not very realistic, and a more precise model like we propose in [23] should be used; however, the $f$-model can be improved by imposing realistic restrictions on the estimates (Section VII). Finally, our conclusions are presented in Section VIII.

\section{Methodology}

The experiments are based on an event-based simulation of EASY scheduling, where events are arrivals and terminations. Upon arrival, the scheduler is informed of the number of processors the job needs and its estimated runtime. It can then start the job's simulated execution or place it in a queue. Upon a job termination, the scheduler is notified and can schedule other queued jobs on the freed processors. Job runtimes are part of the simulation input, but are not given to the scheduler.

Tab. I lists the four traces we used to drive the simulations. These are available through the Parallel Workload Archive [15]. As recommended, we used the sanitized version of the traces [7], [24]. Since these traces span the past decade, were generated at different sites, on machines with different sizes, and reflect different load conditions, we have reason to believe consistent results obtained in this paper are representative. The traces are simulated using the exact data provided, with the possible modification of replacing real user estimates with those generated by the $f$-model, as noted.

Scheduling performance is measured using average wait time and bounded slowdown. The wait time is between a job's submittal and its start time. Slowdown is response time (waittime plus runtime) normalized by runtime. Bounded slowdown eliminates the emphasis on very short jobs (e.g. jobs with zero runtime) due to having the runtime in the denominator. A commonly used runtime lower-bound of 10 seconds was set, yielding the formula: $\max \left(1, \frac{w+r}{\max (10, r)}\right)$, where $w$ and $r$ are the job's wait and run times in seconds, respectively. To reduce warmup effects, the first $1 \%$ of terminated jobs were not included in the results; to reduce cooldown effects, jobs terminating after the last arrival were also not included [10].

Finally, we have chosen f's minimal value to be zero, because this seems to be best aligned with the perception that "zero badness" implies perfect accuracy. However, due to the multiplicative nature of this factor, it is often more convenient to use $F=f+1$. With this, the random model uniformly draws an estimate of a job with runtime $r$ from $[r, r \cdot F]$, and the deterministic model sets the estimate to be $r \cdot F$. Note that in all figures where badness is shown along the $\mathrm{X}$-axis, the random $F$-model is plotted against the deterministic $F / 2$-model, such that both have the same mean.

\section{Performance as a Function of Badness}

Statistical Confidence: The first step we take in trying to uncover the impact of increased "badness" $(=f)$ on performance is to expose the trends underlying the very noisy Fig. 3. To this end we repeat each simulation 100 times with different seeds, and plot the mean of the results ("random") and the and $90 \%$ interval ("90\% confidence", from the 5th percentile to the 95th percentile). Fig. 4 shows that this turns the initial noisy results into relatively smooth curves (at the price of performing about 10,000 simulations of scheduling the jobs in each trace). 

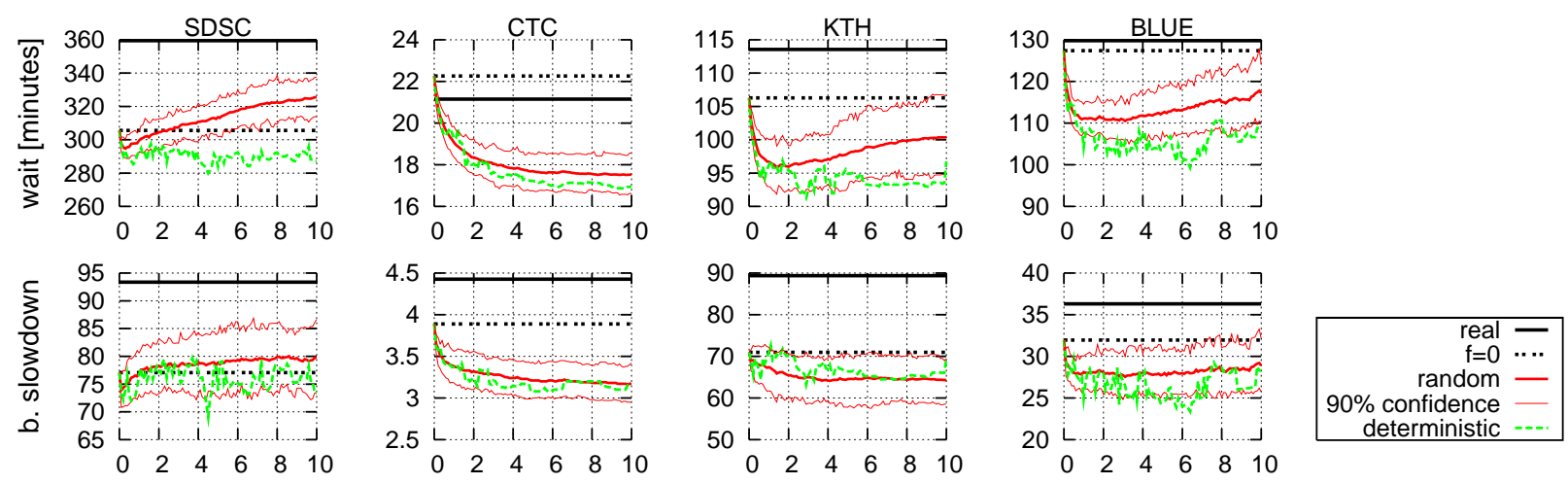

$\mathrm{f}$ (badness factor)

Fig. 4. Statistics from repeated simulations expose clearer performance trends (compare with Fig. 3).

V Trend vs. L Trend: Fig. 4 reveals two trends: ${ }^{2}$ The first is $\mathrm{V}$ shaped (most pronounced for SDSC), and the second is $\mathrm{L}$ shaped (CTC). In both cases, random performance curves initially drop (improve) for small $f$ values. Then, the curves either asymptotically converge to some value ( $\mathrm{L}$ shape), or the trend is first reversed and only then converges ( $\mathrm{V}$ shape). This general tendency continues to larger $f$ values: BLUE is actually $\mathrm{V}$ shaped in both metrics (its curves are quite similar to that of SDSC when changing the $X$ scale to $f \in[0,100]$ and bigger); KTH/wait and KTH/slowdown are L and V shaped, respectively. The deterministic model obviously stays noisy (only one sample per $f$ ), but it is evident that its curves are usually found in the proximity of the lower (better) performance bound of the random model.

If grouping SDSC and BLUE (V shapes only) and comparing them to CTC and KTH (some L shapes), then Tab. I reveals they can be characterized as having higher and lower load, respectively (in this paper the term "load" means "offered load" or "utilization"). This classification was corroborated by simulating all the logs under "high" and "low" load conditions, achieved by artificially multiplying all arrival times by a constant (e.g. if BLUE's original load is $76 \%$, we can raise it to $80 \%$ by multiplying all arrival times by $\left.\frac{76}{80}\right)$.

Expressed in terms of means of multiple simulations, performance is either V or L shaped. Higher or lower average load implies a tendency towards a V or L shape, respectively. The deterministic model is usually closer to the best performance results of the random model.

\section{BACKFILLING AS A FunCTION OF BADNESS}

Holes vs. Balance: Our goal is to understand the reason for the system behavior as reported in Finding 1. A reasonable first step is to validate or disprove the (contradicting) claims underlying the "holes" and "balance" arguments. Though we already know both fail to provide a full explanation to the

\footnotetext{
${ }^{2}$ While not shown due to space limitations, we have also conducted simulations with bigger $f$ values and the results reported here are partly based on these additional experiments.
}

observed performance trends (e.g. the $\mathrm{V}$ shape), determining which argument (if any) better describes the effect of increased $f$ on backfilling is essential. Recall the holes argument implies backfilling activity intensifies with $f$, whereas the balance argument claims the effect of bigger holes evens out by backfill candidates appearing proportionally longer.

Results: Fig. 5 shows the percent of jobs that were backfilled, as a function of $f$, along with their average runtimes. The trends are consistent and the confidence intervals are tight. Backfilling rates clearly increase with $f$ : The exact numbers are workload dependent in that higher loads (Tab. I) imply higher rates. But when simulating the logs under equal high/low load conditions (as in Sec. III), the rates become remarkably similar. The runtimes of backfilled jobs also follow the same pattern, and so do their sizes (not shown).

In accordance to the holes argument and in contrast to the balance argument, bigger $f$ implies more jobs that enjoy backfilling. On average, these jobs are longer and wider.

A possible interpretation of this finding is as support for the L-shaped performance curves (CTC, Fig. 4). This is based on the notion that jobs can be partitioned into "light" or "heavy" based on whether their characteristics allow them to be backfilled or not, and it seemed that bigger $f$ simply means that more jobs become "light" and can enjoy better service. However, as we will show below, our finding doesn't just mean "more" jobs. It can also mean different jobs, and specifically longer jobs, possibly at the expense of shorter ones.

\section{The Heel-And-Toe Dynamics}

Heel-and-Toe Hypothesis: The question that follows Finding 2 is why is it so? What's wrong with the balance argument? Why isn't the effect of bigger holes canceled by the backfill candidates that are proportionally longer? After reexamining the backfilling rules, we came up with a possible explanation, as illustrated in Fig. 6. To simplify, assume all estimates are exactly double the runtime ( $F=2$ under the deterministic model as defined in Sec. II). Based on the information available to the scheduler at $T_{0}$ (time 0 ), it appears the earliest time for 

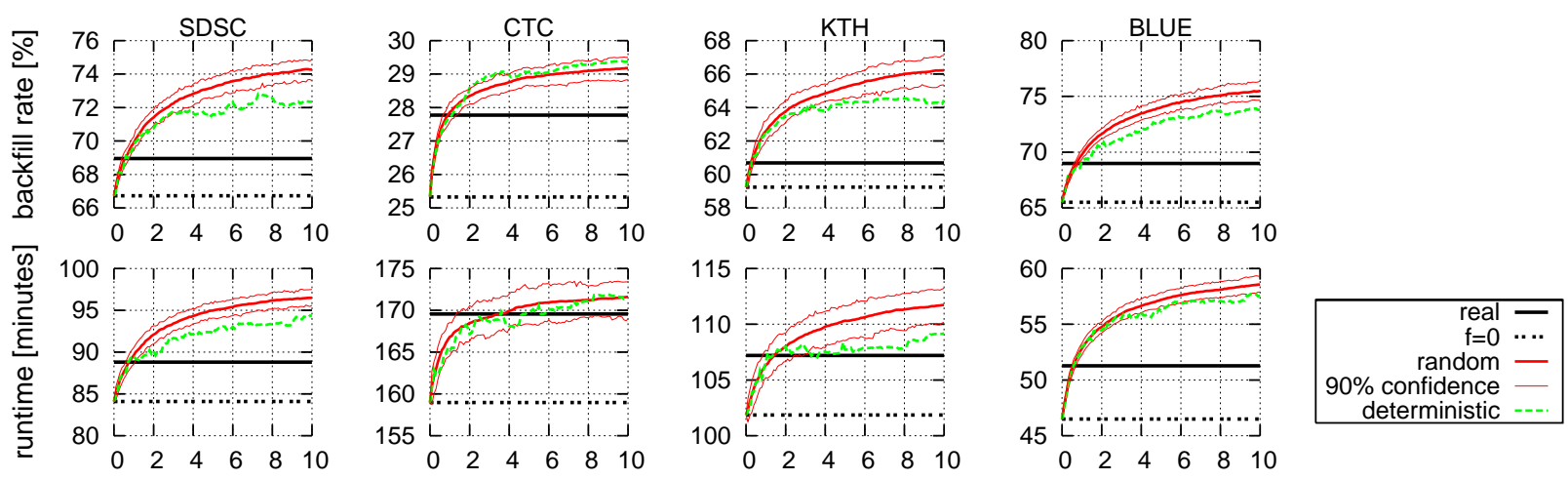

$\mathrm{f}$ (badness factor)

Fig. 5. The percent of backfilled jobs and their average runtimes monotonically increase with $f$. In all cases, the relative increase is roughly similar, e.g. the rates/runtimes associated with $f=10$ are $10-20 \%$ bigger than that of $f=0$.

$J_{3}$ (job 3) to start is $T_{12}$, even though the real earliest start time is actually $T_{6}$. Thus, the scheduler makes a reservation on $J_{3}$ 's behalf for $T_{12}$ and can only backfill jobs that honor this reservation. At $T_{4}, J_{2}$ terminates. As $J_{1}$ is still running, nothing has changed with respect to $J_{3}$ 's reservation, and so the scheduler scans the wait queue in search of appropriate candidates for backfilling. $J_{4}$ (the first backfill candidate under FCFS) fits the gap between $T_{4}$ and the reservation $\left(T_{12}\right)$ and so it is backfilled, effectively pushing back the real earliest time at which $J_{3}$ could have started from $T_{6}$ to $T_{8}$.

SJFness: This "heel-and-toe" scenario, of repeatedly pushing away the earliest starting point of the first queued job, step by step, may continue until $T_{12}$ is reached. During this time, the window between the current time and the reservation time is continuously shortened, such that waiting jobs that fit this open gap get shorter and shorter, effectively nudging the system towards Shortest-Job First (SJF) scheduling. (Note that the initial open gap can be very short to begin with.) And so, if the heel-and-toe dynamic does in fact occur, this limited form of "SJFness" contributes to the performance improvement reported in Finding 1, namely, the first (descending) part of the V-curves, and the L-curves in their entirety. This effect is directly quantified in the next section.

Tendency towards SJFness with positive $f$ was also observed (but not explained) by Zotkin and Keleher [28], which conducted an "off-line" simulation of what happens when all the jobs in a trace arrive at the same exact time instance. They found that, in comparison to $f=0$, shorter jobs leave the system at a faster rate when estimates are set to be five times the actual runtime. The heel-and-toe dynamics explain this phenomenon.

Verifying Heel-and-Toe Occurs: Let $J_{h}$ be the first queued job (meaning $J_{h}$ isn't backfilled, but rather, it waits for its turn, becomes first, and gets a reservation). Let $S_{h}$ denote the real shadow time of $J_{h}$, defined to be $J_{h}$ 's (hypothetical) starttime, if all estimates suddenly become completely accurate. For example, the initial real shadow of $J_{3}$ in Fig. 6 is $T_{6}$. During the time $J_{h}$ is first, we say that a backfill operation is wild if it causes $S_{h}$ to be delayed, or that it's mild, otherwise. All the backfill operations in Fig. 6 are wild, because all resulted in a change of the real shadow. By definition, showing that wild backfilling happens means proving that heel-and-toe dynamics indeed occur. Fortunately, detecting wild backfilling is easy in a simulation: We compute $S_{h}$ by traversing the run-list in (real) termination order and finding the earliest time in which enough free processor accumulate to satisfy $J_{h}$. By doing this before/after a backfill operation, we can tell if the operation is wild ( $S_{h}$ changed) or mild (stayed the same).

Fig. 7 clarifies that the heel-and-toe dynamic is not just hypothetical, e.g. with $f=10,2-5 \%$ of the jobs are wildly backfilled. The X-axis doesn't start at zero, because there can be no wild backfilling with perfect estimates. The consequences of wild backfilling are delayed jobs that suffer from at least one wild backfill operation while they are at the head of the queue (as $J_{3}$ in Fig. 6). Fig. 8 (top) shows that around $1 \%$ of the jobs are delayed. Any performance improvement obtained by the $f$-model is at the expense of these jobs. The bottom of Fig. 8 shows the average delay duration. This is the elapsed time between $J_{h}$ 's initial real shadow and its eventual start time (the "stolen" time in Fig. 6), and it grows with $f$. The average number of times $S_{h}$ is delayed also grows with $f$ (not shown).

Holes vs. Balance Revisited: Our findings indicate that the seemingly contradictory "balance" and "holes" arguments can in fact be reconciled: The performance improvement attributed to positive $f \mathrm{~s}$ is not just because of wider holes in the schedule that allow for more backfilling (in accordance to the "holes" argument), because backfill candidates are indeed widened proportionally (in accordance to the "balance argument"). Rather, it is the result of a heel-and-toe effect that manages to keep the holes open by backfilling shorter jobs, which repeatedly delay the execution of the first queued job and lead to an SJF-like schedule.

The heel-and-toe dynamic (1) is verified to occur in practice, (2) reconciles between the balance and holes arguments, and (3) leads to a limited form of SJFness. Thus, it explains the performance improvement due to positive $f$ values.

Let us now explain why performance can also become worse. 

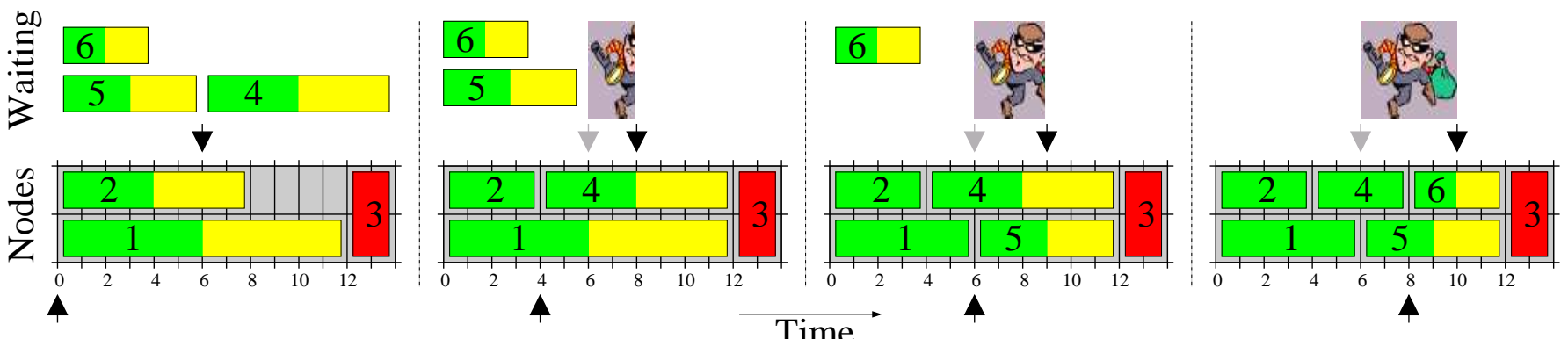

Fig. 6. Illustrating heel-and-toe. Job numbers indicate arrival order. Job estimates are exactly double their runtime $(F=2)$. The left portion of jobs (green/dark) indicates their real runtimes. Due to the doubling, the scheduler views jobs as twice as long (right portion; yellow/bright). The bottom arrows show the progress of time, whereas the top black arrows show the earliest time at which job 3 would have been started, had real runtimes been known (at that particular point in time). The thief's width shows the amount of "stolen" time, at the expense of job 3.
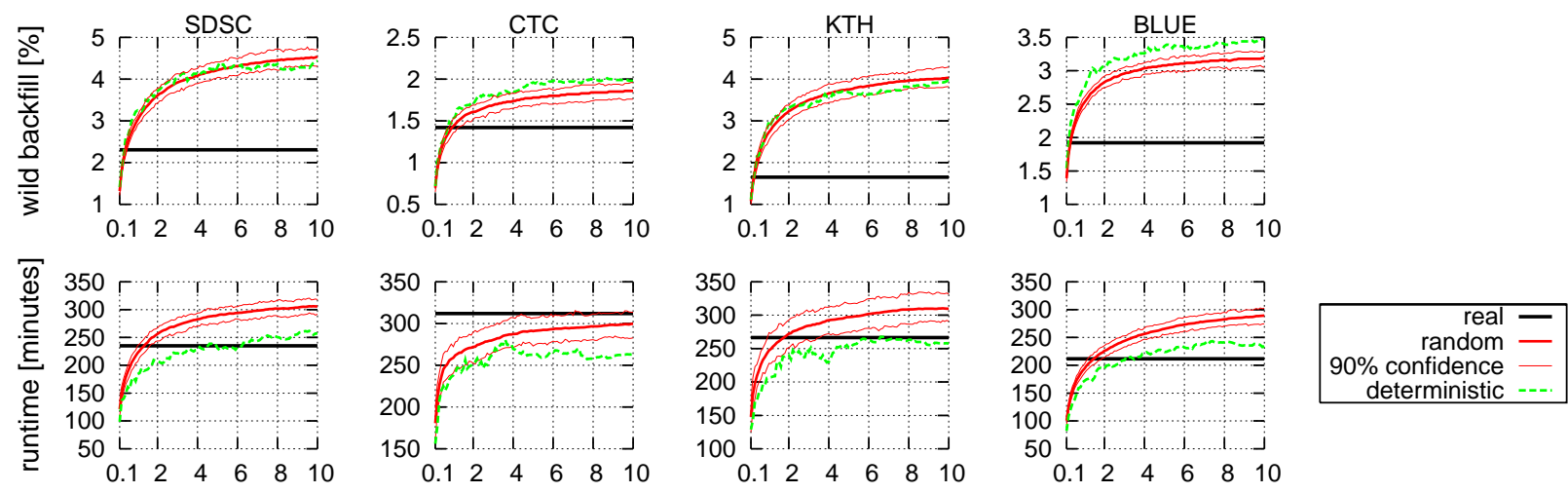

$\mathrm{f}$ (badness factor)

Fig. 7. Existence of wild backfilling demonstrates heel-and-toe dynamics occur. The rate of wild jobs and their average runtime follow the same trends as in the general case (Fig. 5).
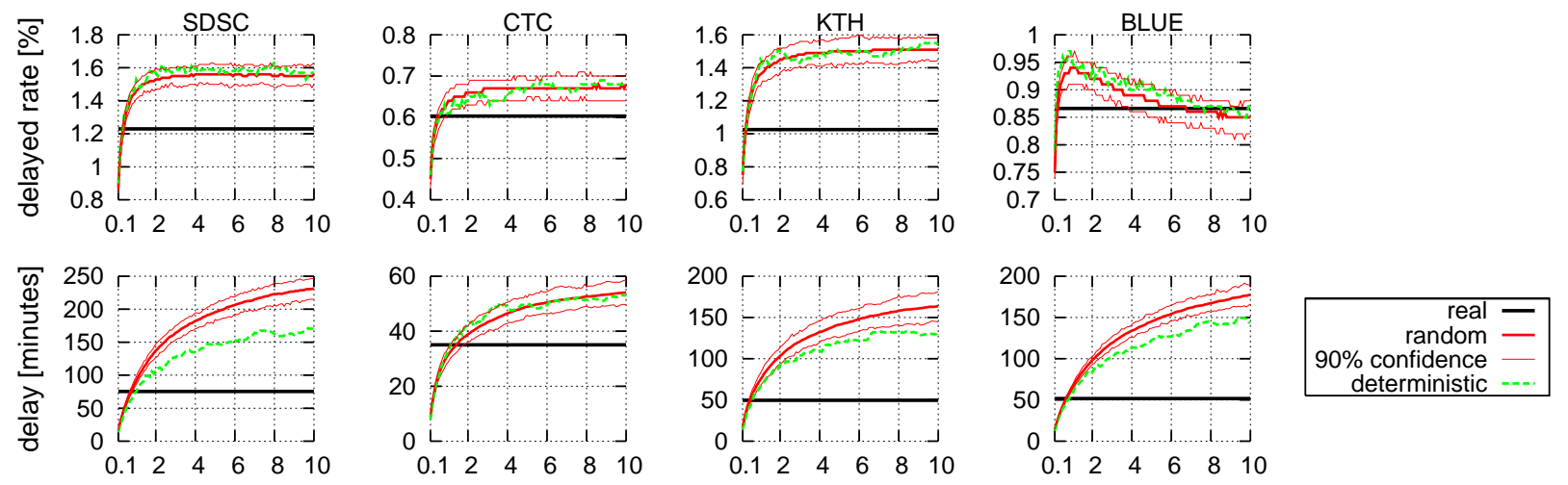

$\mathrm{f}$ (badness factor)

Fig. 8. The percentage of jobs that suffer from wild backfilling (top), and the average delay (bottom). The unique trend observed in BLUE (top) is also displayed by the other logs if simulating high load conditions as in Sec. III and examining a slightly wider $f$ range; on the other hand, BLUE becomes like all the others if simulating low load conditions.

\section{Countering the SJFness of HeEl-And-ToE}

We now focus on the second, ascending, part of the V-shaped performance curves where performance continuously degrades (Finding 1; Fig. 4). The explanation has two components: the increased $f$, and the resulting amplification of randomness (for the non-deterministic model). These components increasingly counteract the SJFness reported earlier.
Increased $f$ : As shown in Fig. 5 (and highlighted in Finding 2), backfilling activity monotonically increases with $f$, while at the same time, the runtime of backfilled jobs becomes longer. Longer average runtimes wouldn't be problematic by themselves, had short jobs nevertheless been prioritized. But this is not the case. To illustrate why, let us reconsider the scenario depicted in Fig. 6. Tab. II lists the estimates of jobs at time $T_{4}$ (after $J_{2}$ terminates) for various $F$ values, as well as 

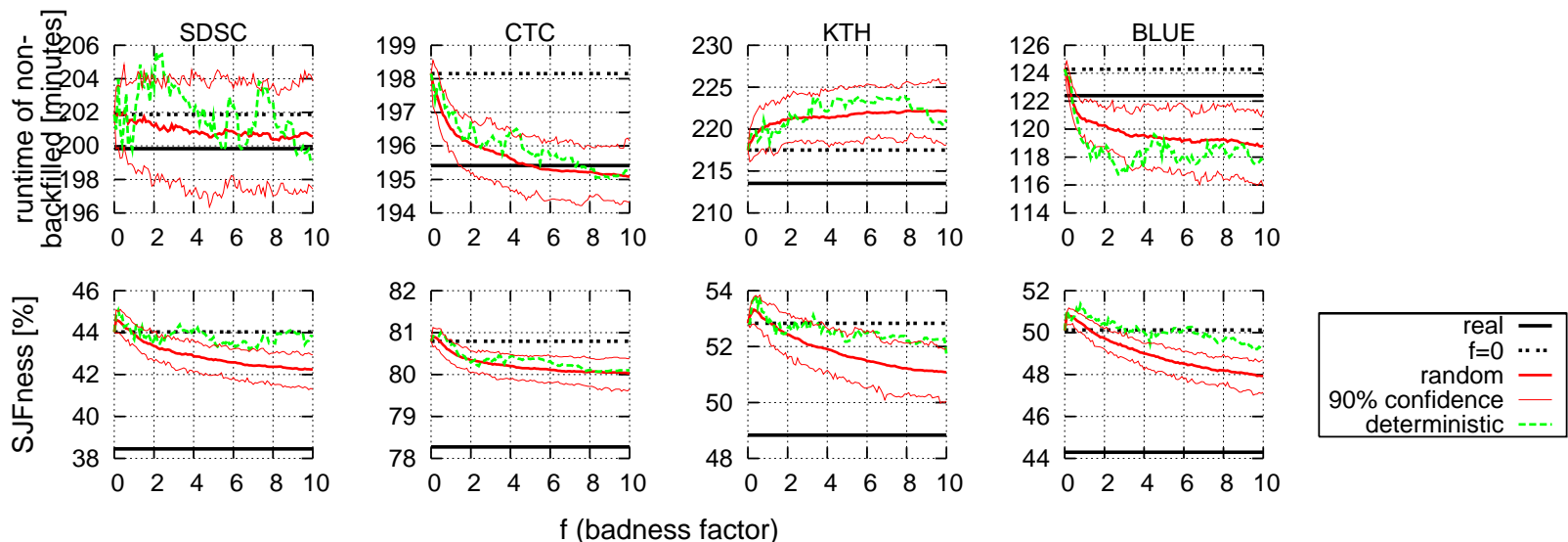

$\mathrm{f}$ (badness factor)

Fig. 9. Average runtime of non-backfilled jobs is usually made shorter when increasing $f$ (top). Average SJFness initially rises, but there's a quick trend change as backfilled jobs become longer.

\begin{tabular}{|c||c|c|c|c||c|}
\hline \multicolumn{1}{|c||}{$F$} & \multicolumn{4}{c||}{ estimates } & hole length \\
\cline { 2 - 5 } & $J_{1}$ & $J_{4}$ & $J_{5}$ & $J_{6}$ & at $T_{4}$ \\
\hline \hline 1 & 6 & 4 & 3 & $\mathbf{2}$ & 2 \\
$1 \frac{1}{3}$ & 8 & $5 \frac{1}{3}$ & $\mathbf{4}$ & $2 \frac{1}{3}$ & 4 \\
2 & 12 & $\mathbf{8}$ & 6 & 4 & 8 \\
\hline
\end{tabular}

TABLE II

The length of the hole in the schedule and the estimates of jobs in

Fig. 6, for various $F$ values, at time $T_{4}$. In the first row $F=1$ so estimates equal runtimes; in subsequent rows estimates are runtimes multiplied by $F$. For each $F$, the estimate of the first job that fits the hole appears in bold.

the length of the resulting hole. The last row simply specifies what is shown in Fig. $6(F=2)$. Recall that job indexes indicate arrival order, used by the scheduler when searching for backfill candidates. Thus, $J_{4}$ is the first candidate and since it fits the existing hole it is chosen for backfilling. However, if the value of $F$ had been $1 \frac{1}{3}$ instead of 2 (second row in Tab. II), then the hole would have been proportionally smaller and the scheduler would have deemed $J_{4}$ as too long for backfilling, favoring instead the shorter $J_{5}$ for execution. If $F$ was further reduced to 1 (complete accuracy; first row), than $J_{5}$ would also appear as too long, effectively making $J_{6}$ (the shortest waiting job) the only eligible candidate. We can therefore see there's a subtle tradeoff here:

While bigger $f$ means more backfilling (which short jobs enjoy more than longer ones), the bigger holes do in fact allow longer jobs to backfill.

This finding is verified in Fig. 9. First, the top row shows the average runtime of non-backfilled jobs: this usually becomes shorter with increased $f$, suggesting the scheduler indeed makes "wrong" decisions by forcing shorter jobs to wait and preferring longer jobs for backfilling (Fig. 5). More important is the bottom row that directly quantifies the effect: "SJFness" is the percent of jobs that are the shortest in the waiting queue at the time they are chosen to run. Evidently, SJFness intensifies with very small $f$ values, only to monotonically drop later on (perfectly coinciding with our explanation above).

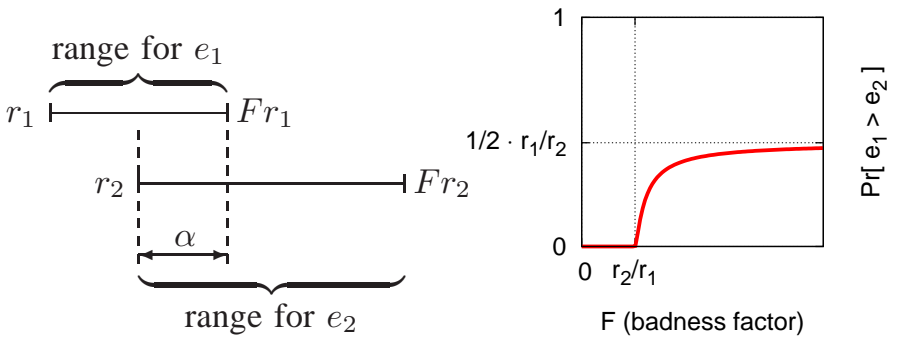

Fig. 10. Probability that $e_{1}>e_{2}$ when actually $r_{1}<r_{2}$; see text for details.

Increased Randomness: The situation gets worse when randomness is introduced, as now, in addition, long jobs can masquerade as short jobs and vice versa. To illustrate this, let $J_{1} / J_{2}$ be two jobs within the wait queue with runtimes $r_{1} / r_{2}$ and estimates $e_{1} / e_{2}$ that were generated by the random $f$ model, respectively. This is depicted in Fig. 10 (left), assuming $r_{1}<r_{2}$ without loss of generality. We are interested in $\operatorname{Pr}\left(e_{1}>e_{2}\right)$, that is, the probability the scheduler is erroneously told that $J_{1}$ is longer than $J_{2}$. By conditioning (Bayes' theorem) this is

$$
\begin{aligned}
\operatorname{Pr}\left(e_{1}>e_{2}\right)= & \operatorname{Pr}\left(e_{1}>e_{2} \mid e_{1}, e_{2} \in \alpha\right) \cdot \operatorname{Pr}\left(e_{1}, e_{2} \in \alpha\right)+ \\
& \operatorname{Pr}\left(e_{1}>e_{2} \mid \overline{e_{1}, e_{2} \in \alpha}\right) \cdot \operatorname{Pr}\left(\overline{e_{1}, e_{2} \in \alpha}\right)
\end{aligned}
$$

where $\alpha \equiv\left[r_{2}, F r_{1}\right]$ is the intersection of the two ranges from which $e_{1}$ and $e_{2}$ are drawn. The second term in the above summation is obviously zero (when either $e_{1}$ or $e_{2}$ are outside $\alpha$ then $e_{1}<e_{2}$ ) and so we are left with

$$
\begin{aligned}
& \operatorname{Pr}\left(e_{1}>e_{2}\right)= \\
& \underbrace{\operatorname{Pr}\left(e_{1}>e_{2} \mid e_{1}, e_{2} \in \alpha\right)}_{\lambda_{1}} \cdot \underbrace{\operatorname{Pr}\left(e_{1} \in \alpha\right)}_{\lambda_{2}} \cdot \underbrace{\operatorname{Pr}\left(e_{2} \in \alpha\right)}_{\lambda_{3}}
\end{aligned}
$$

If $\alpha$ exists $\left(F r_{1}>r_{2}\right)$, then $\lambda_{1}=\frac{1}{2}$, because it's simply the probability one number is bigger than another if both are uniformly chosen from the same range. (If $\alpha$ is degenerate then $\lambda_{1}=0$.) As $\lambda_{2}$ and $\lambda_{3}$ represent standard events in a uniform setting, we get 

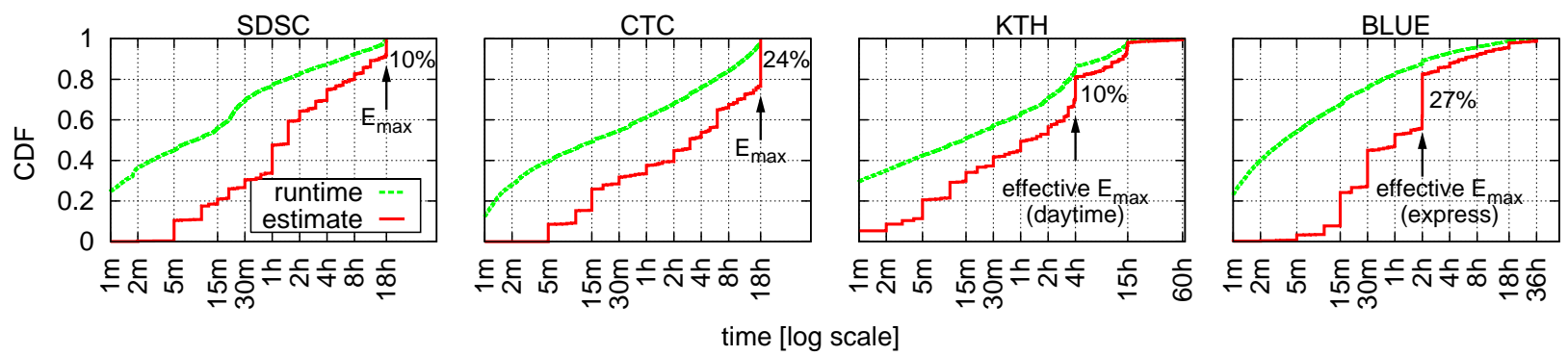

Fig. 11. Cumulative distribution function (CDF) of runtimes and estimates. Unlike runtimes, estimates are modal. Runtime curves appear higher because runtimes are always shorter than estimates (underestimates jobs are killed).

$$
\begin{aligned}
\operatorname{Pr}\left(e_{1}>e_{2}\right) & =\frac{1}{2} \cdot \frac{F r_{1}-r_{2}}{F r_{1}-r_{1}} \cdot \frac{F r_{1}-r_{2}}{F r_{2}-r_{2}} \\
& =\frac{1}{2} \cdot \frac{F^{2}}{(F-1)^{2}} \cdot \frac{r_{1}}{r_{2}}+\mathrm{O}\left(\frac{1}{F}\right)
\end{aligned}
$$

thus the error probability is monotonically increasing and converges to $\frac{1}{2} \cdot \frac{r_{1}}{r_{2}}$ when $F$ goes to infinity (Fig. 10 right).

Under the random model, the bigger the $f$, the more it is
probable the scheduler would erroneously view short jobs
as long and vice versa. This explains why SJFness is higher
for the deterministic model (Fig. 9) and hence why the
deterministic model consistently outperforms the random
model (Fig. 4).

VII. Making the Model More Realistic

The Problem: The $f$-model is the dominant model for generating artificial user runtime estimates and evaluating the impact of inaccurate user estimates on backfilling algorithms [20], [28], [14], [1], [18], [27], [16], [3], [9]. Based on the $f$ model, researchers have drawn neat conclusions that range from "performance is independent of accuracy" to "inaccuracy actually improves performance". Indeed, when employing artificial estimates as generated by the $f$-model, these claims may reflect certain aspects of the truth, as shown above. However, there is a fundamental problem with all the insights that are based on the $f$-model:

THE PROBLEM WITH THE $f$-Model
Increased inaccuracy as modeled by larger $f$ values effec-
tively spreads the estimates across a larger range. But with
real estimates it's exactly the opposite! Namely, inaccuracy
manifests itself by more jobs using the same estimate value.
Thus, conclusions based on the theoretical $f$-model might
not apply when real user estimates are involved.

Understanding results that are based on the $f$-model can be interesting and important. For example, the heel-and-toe dynamics turned out to be the reason why, as shown in Fig. 2, doubling of real user estimates improves performance (doubling is a legitimate scheduling optimization, and there are other related practical issues [22]). Nevertheless, such understandings can have only limited applicability to real systems that employ real user estimates. Importantly, a statement like "inaccuracy improves performance" is a misleading oversimplification: real inaccuracy is actually tightly correlated with degraded performance, as will be exemplified next.

Modality of Real Estimates: Human users don't choose estimates that are uniformly distributed between the real runtime and its multiple with some value. Instead, they use arbitrarily "round" estimates, e.g. 5 minutes, 1 hour, etc. In fact, we found that about $90 \%$ of the jobs repeatedly use the same 20 "round" values [23]. This modality is reflected in the staircase-like CDF curves shown in Fig. 11, where each mode corresponds to a popular estimate. One particular value that is especially popular is $E_{\max }$, the maximal estimate allowed. This is a derivative of the per-site administrative upper bound on runtimes, which may differ from site to site, but is always enforced. The value of $E_{\max }$ is typically around $18 \mathrm{~h}$; in KTH and BLUE $4 \mathrm{~h}$ and $2 \mathrm{~h}$ serve as the "effective" $E_{\max }$ because most jobs were submitted during daytime or to the interactive/express queues, respectively. $E_{\max }$ is used by $10-27 \%$ of the jobs and is the most popular in three of the four logs (in SDSC it's ranked third). Its immense popularity can probably be attributed to users who want to "play it safe" and prevent their jobs from being prematurely killed.

Implications of Modality: Regardless of the reason for $E_{\text {max }}$ 's popularity, the implications are dire in terms of performance. To understand why, consider an extreme case in which all jobs use $E_{\max }$ as their estimate. The outcome is that all backfilling activity, as shown in Fig. 1, completely stops: The reservation of the first queued job is computed based on estimated termination times of currently running jobs, and these will all occur before $E_{\max }$ time, by definition. Hence, the reservation itself will occur before $E_{\max }$ time and therefore backfilling holes (from the present time until the reservation) are always smaller than $E_{\max }$. Since we assume all estimates of waiting jobs are exactly $E_{\max }$, none of them will fit the holes in the schedule. Thus scheduling largely reverts to plain FCFS, which result in a serious performance degradation. (The only remaining backfill activity is using the "extra" nodes [13]).

Surprisingly, the mere existence (and popularity) of $E_{\max }$ is completely overlooked by researchers. For example, Cirne and Berman conjectured that the apparent connection between longer runtimes and increased accuracy, shown in Fig. 12, 


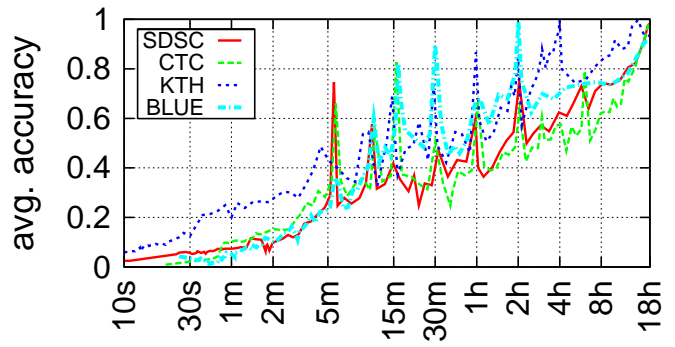

avg. runtime [log]

Fig. 12. Average accuracy ( $\left.\frac{\text { runtime }}{\text { estimate }}\right)$ as a function of runtime. (Xaxis groups jobs to 100 equally-sized bins according to their runtime.)

is because the more a job progresses in its computation, the grater its chances become to reach successful completion [2]. Such assumptions are unwarranted, as the existence of $E_{\max }$ guarantees long jobs to have high accuracy: for example, assuming $E_{\max }$ is $18 \mathrm{~h}$, if a job's runtime is $17 \mathrm{~h}$, then its estimate must be between $17 \mathrm{~h}-18 \mathrm{~h}$ and thus at least $94 \%$ accurate. In other words, long jobs are on the right of Fig. 12, where accuracy is high, while short jobs tend to be on the left, at lower accuracies.

The peaks in Fig. 12 are due to popular estimates (smaller than $E_{\max }$ ) and the many underestimated jobs that used them and are subsequently killed. But many other jobs that use these popular values are in fact significantly overestimated. The problem is that in a real system the scheduler has no way to distinguish between such jobs. In contrast, when the $f$-model is used, the scheduler actually gets pretty good information. To clarify, consider a scheduler that explicitly favors shorter jobs for backfilling [28], [1], [22] and must work with inaccurate estimates. Such a scheduler does not really need to know precise runtimes - it just needs to know the correct relative ordering, and the $f$ model provides the correct ordering with a probability of at least $1-\frac{r_{1}}{2 r_{2}}$. This can dramatically improve performance (up to an order of magnitude according to [1]). As shown earlier, heel-and-toe dynamics nudge a FCFS-based scheduler towards SJFness, and therefore the same argument applies. Further, an estimate distribution that is dominated by only a few distinct modes ( $E_{\max }$ and others) negatively effects performance, because less variance among waiting jobs means less opportunities for the scheduler to exploit existing holes (with various sizes) for backfilling.

Enforcing an Upper Bound on Estimates: The bottom line is that if one wants to model increasing user inaccuracy, one should focus on the modality of user estimates. For example, $10 \%$ of the jobs using $E_{\max }$ is an optimistic scenario relative to $20 \%$, which in turn is more optimistic than $30 \%$. Modeling increased inaccuracy by gradually associating more jobs with $E_{\max }$ is more realistic than using the vanilla $f$-model. Fortunately, $E_{\max }$ can be naturally incorporated within the $f$ model, by truncating estimates that come out too high: if the model estimate is $e$, we instead use $\min \left(e, E_{\max }\right)$. Let this be denoted as the truncated $f$-model. This model has the property that bigger $f$ values imply more jobs associated with $E_{\max }$.

Fig. 13 shows the results. The truncation has negligible impact for very small $f$ values, because at this points very few artificial estimates exceed $E_{\max }$. The common trend is therefore of improved performance, similarly to the vanilla $f$ model. Truncation gradually becomes the dominant factor as $f$ increases and so the trend is reversed. The difference between the truncated (Fig. 13) and vanilla (Fig. 4) models when $f$ goes to infinity is that the ascending part of the latter never intersects the curves associated with real user estimates (except BLUE/wait, verified till $f=10,000$ ), whereas the former always does. At the intersection point, the truncated model is successful in "capturing the badness" of the real estimates. Thus, with big enough $f$, the behavior of the truncated model coincides with our claim above that performance degrades if inaccuracy is increased by making the estimate distribution more modal.

An Accurate Model: While the truncated $f$-model is more realistic than the vanilla one, its output is still fundamentally different from real estimates. A key difference is that only one mode is created (at $E_{\max }$ ), whereas real estimates exhibit several modes (Fig. 11). Further, the $E_{\max }$ mode as created by the model is poorly constructed: it consists of long jobs only (with big enough runtimes such that multiplying them with $F$ results in estimates bigger than $\left.E_{\max }\right)$. In reality, many short jobs are estimated by users to run $E_{\max }$. Of these, most notable are jobs that fail on startup. Thus, even with the truncated model, the scheduler can still identify shorter jobs better than when real estimates are employed.

These reasons lead to the undesirable situation where each trace/metric combination requires a different $f$ to obtain performance results comparable to those of real estimates (crossovers in Fig. 13). This serious drawback is contrasted with the model's simplicity and ease of implementation and use. We therefore view it as the "quick and dirty" substitute for the vanilla version, namely, if faced with the choice of using either one of them, we strongly support the truncated version. It is our opinion that while it is not perfect, it is also not "garbage".

In general, however, we advocate using the more sophisticated estimate model we have developed in [23], instead of the $f$-model variants. This paper serves in part as motivation. The input of our new model is $E_{\max }$ and optionally the percent of associated jobs. ${ }^{3}$ The optional argument allows to gradually increase inaccuracy in a truly realistic manner. The output of the new model is a series of modes, where each mode is a pair consisting of an estimate value and the percent of jobs that use it (twenty of which cover $90 \%$ of the jobs). This means that in contrast to common practices, estimates are not generated on a per-job basis, but collectively. Thus, our model also provides a way to map the generated distribution onto a set of jobs with predetermined runtimes, such that each job's assigned estimate is equal to or bigger than its runtime, as required by the backfilling rules. The model is available for download at [21], and was verified to produce results that are almost identical to the real thing [23].

\footnotetext{
${ }^{3}$ We show that the dissimilarity between estimate distributions of different traces is largely embodied in the percent of jobs that use $E_{\max }$ as their estimate; the distributions are otherwise remarkably similar.
} 

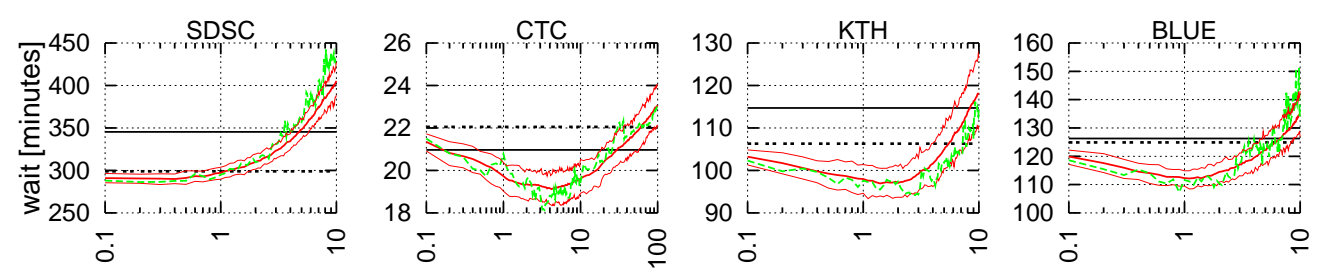

\begin{tabular}{|l|r|r|}
\hline \multirow{2}{*}{ trace } & \multicolumn{2}{|c|}{ intersection $f$} \\
\cline { 2 - 3 } & wait & \multicolumn{1}{|c|}{ b. sid } \\
\hline SDSC & 4.77 & 24.50 \\
CTC & 29.82 & 376.09 \\
KTH & 8.36 & 124.35 \\
BLUE & 6.68 & 47.63 \\
\hline
\end{tabular}
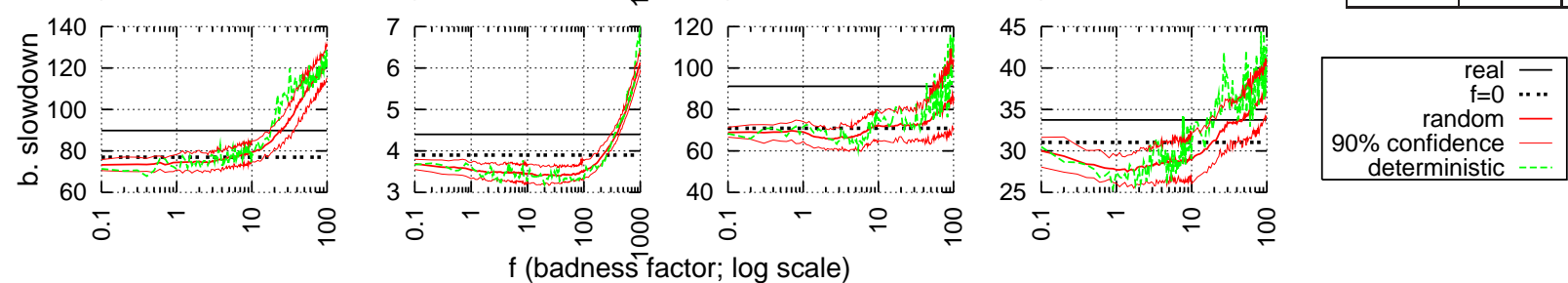

Fig. 13. Performance results obtained with the truncated $f$-model (compare with Fig. 4). The table specifies the intersection point between curves associated with the "random" model and those associated with real estimates. (Slight differences exist between results associated with real user estimate of the vanilla and the truncated models. This is due to runtimes bigger than $E_{\max }$ that unexplainably exist in the original logs and were truncated to make sure they are not bigger than the associated estimates.)

\section{CONCLUSIONS}

User runtime estimates are required for backfilling, currently the most popular scheduling scheme of parallel systems. Many studies have claimed that system performance is either unaffected by, robust to, or improves with increasingly inaccurate estimates. The de-facto standard model for obtaining such results has been the $f$-model that given a runtime $r$, uniformly chooses the associated estimate from $[r,(f+1) \cdot r]$, or deterministically sets it to be $(f+1) \cdot r$. With this model, bigger $f \mathrm{~s}$ imply increased inaccuracy. Studies that reported a performance improvement explained it with the "holes" argument, claiming that increased overestimation of long jobs opens larger holes in the schedule for backfilling shorter jobs. In contrast, studies reporting performance is unaffected have used the "balance" argument, claiming the larger holes cancel out by the fact backfill candidates appear proportionally longer.

We found performance is extremely sensitive to minor changes in $f$, and that within the noisy results space the contradictory observations about performance trends are both possible, when using only few samples in a non-systematic manner. However, averaging over repeated simulations revealed that the mean effect of increasing $f$ is usually $\mathrm{V}$-shaped: average wait time and slowdown drop at low inaccuracies and the trend is gradually reversed for larger $f \mathrm{~s}$ (though large $f$ s still yield better results than $f=0$ ). To explain this, we show that the seemingly contradictory "balance" and "holes" arguments are both incorrect, or rather, correct to some extent, but miss the key issue that reconciles between them. Performance improvement due to increased $f$ is not simply the result of more backfilling due to more holes in the schedule. Rather, it is the result of a "heel-and-toe" dynamic: a distinctive sequence of events where small backfill jobs continuously prevent the holes from closing $u p$, leading to a preference for short jobs and the automatic production of an SJF-like schedule. When $f$ is very small, the proportionally narrow holes make sure only jobs that are truly short enjoy the effect (explains the initial descending part of the $\mathrm{V}$-shape). However, as $f$ gets bigger, increasingly longer jobs can enjoy it too (explains the ascending part). The situation is worse for the random model, which allows long jobs to masquerade as short and vice versa (explains why the deterministic model yields better performance). We have directly quantified this by measuring the "SJFness" as a function of $f$, defined to be the percent of jobs that are the shortest in the wait-queue at the time they are started. The result was consistently $\Lambda$-shaped, a kind of mirror image to the $\mathrm{V}$ performance curves.

Fully understanding the $f$-model highlights its fundamental flaw: it leads to a limited SJF-like scheduling, and indeed, SJF is insensitive to multiplying runtimes by some factor as long as the relative ordering of jobs is preserved. But real user estimates provide no such ordering! Rather, they are inherently modal, with $90 \%$ of the jobs using only 20 "round" estimate values (e.g. 1 hour) and, in particular, $10-27 \%$ using $E_{\max }-$ the maximal allowed. Any popular estimate is bad for backfilling, as the scheduler can't differentiate between the associated jobs, e.g. they can have $0 \%$ accuracy (zero runtime) if they fail on startup, $100 \%$ accuracy if they are underestimated and killed by the system, or anything in between if they reach successful completion. However, $E_{\max }$ is especially bad, as the associated jobs appear too long for backfilling, and the more jobs there are that use it, the more the schedule resembles plain FCFS.

The bottom line is that the popular claim that "increasingly inaccurate estimates improve performance" is only correct if "inaccurate" means "multiplied by a factor" (as in the $f$-model), which is far from the truth when real estimates are involved. Inaccuracy of real estimates manifests itself in the form of modality, and "increasing it" means making estimates more modal (e.g. by adjusting the number of jobs associated with $E_{\max }$ from $10 \%$ to $20 \%$ ). In this case, increased inaccuracy actually degrades performance, as one would intuitively expect. Put another way, this paper refutes the overwhelmingly accepted myth that inaccuracy improves (or doesn't effect) performance, on the grounds that it is based on false assumptions. As a consequence, it motivates the quest for deriving and using 
more accurate estimates [22].

We demonstrate the correctness of our findings by suggesting the truncated $f$-model, which adjusts an estimate $e$ that is generated by the vanilla $f$-model to be $\min \left(E_{\max }, e\right)$. This creates a mode at $E_{\max }$, such that bigger $f$ s imply more jobs associated with $E_{\max }$. Indeed, one can "manufacture" arbitrarily bad performance results by choosing a big enough $f$. Importantly, one can always find an $f$ for which results obtained when using artificial estimates are equal to those obtained when real estimates are employed, in contrast to the vanilla model. We view the truncated model as a simple "quick and dirty" substitute for the original $f$ model, and contend it should always be preferred over the latter.

Regrettably, the truncated model is still not realistic. For example, it generates only one mode (at $E_{\max }$ ) and only associates long jobs with it, whereas with real estimates there are several modes and short jobs are associated with all of them. One consequence was that each trace/metric combination required a significantly different $f$ in order to obtain results comparable to those of the real thing. We therefore advocate the use our accurate estimates model as suggested in [23], which was verified to produce results that are remarkably similar to the real thing (both in terms of the estimate distribution and the resulting performance). This model directly targets the modal nature of estimates and allows to gradually increase inaccuracy in a truly realistic manner. It is available for download at [21].

Finally, we note our results have a practical value for scheduling: heel-and-toe dynamics happen also with real user estimates, explaining why doubling them improves performance. In this context, future work includes the evaluation of estimates' effect on fairness - who pays for the average improvement in performance, and how much, if e.g. all (real) estimates are doubled. We also intend to check whether our findings apply to backfill schedulers with an explicit SJF component (such as those proposed in [1]) and see whether the heel-and-toe dynamics work there as well.

Acknowledgments: This research was supported in part by the Israel Science Foundation (grant no. 167/03). Many thanks are due to the people and organizations who deposited their workload logs in the Parallel Workloads Archive.

\section{REFERENCES}

[1] S-H. Chiang, A. Arpaci-Dusseau, and M. K. Vernon, "The impact of more accurate requested runtimes on production job scheduling performance". In 8th Job Scheduling Strategies for Parallel Processing, pp. 103-127, Springer-Verlag, Jul 2002. LNCS 2537.

[2] W. Cirne and F. Berman, "A comprehensive model of the supercomputer workload". In 4th Workshop on Workload Characterization, Dec 2001.

[3] D. England, J. Weissman, and J. Sadago-pan, "A new metric for robustness with application to job scheduling". In 14th IEEE Int'l Symp. High Performance Distributed Comput., pp. 135-143, Jul 2005.

[4] Y. Etsion and D. Tsafrir, A Short Survey of Commercial Cluster Batch Schedulers. Technical Report 2005-13, The Hebrew University of Jerusalem, May 2005.

[5] D. G. Feitelson, "Experimental analysis of the root causes of performance evaluation results: a backfilling case study". IEEE Trans. Parallel \& Distributed Syst. 16(2), pp. 175-182, Feb 2005.

[6] D. G. Feitelson and A. Mu'alem Weil, "Utilization and predictability in scheduling the IBM SP2 with backfilling". In 12th IEEE Int'l Parallel Processing Symp., pp. 542-546, Apr 1998.
[7] D. G. Feitelson and D. Tsafrir, "Workload sanitation for performance evaluation”. In IEEE Int'l Symp. Performance Analysis of Syst. \& Software, pp. 221-230, Mar 2006.

[8] E. Frachtenberg, D. G. Feitelson, F. Petrini, and J. Fernandez, "Adaptive parallel job scheduling with flexible coscheduling". IEEE Trans. Parallel \& Distributed Syst. 16(11), pp. 1066-1077, Nov 2005.

[9] F. Guim, J. Corbalán, and J. Labarta, Impact of Qualitative and Quantitative Errors of the Job Runtime Estimation in Backflling Based Scheduling Policies. Technical Report, Computer Architecture Department, Technical University of Catalonia, 2006.

[10] R. Jain, The Art of Computer Systems Performance Analysis. John Wiley \& Sons, 1991.

[11] J. Krallmann, U. Schwiegelshohn, and R. Yahyapour, "On the design and evaluation of job scheduling algorithms". In Job Scheduling Strategies for Parallel Processing, pp. 17-42, Springer Verlag, 1999. LNCS 1659.

[12] C. B. Lee, Y. Schwartzman, J. Hardy, and A. Snavely, "Are user runtime estimates inherently inaccurate?". In 10th Job Scheduling Strategies for Parallel Processing, pp. 253-263, Springer-Verlag, Jun 2004. LNCS 3277.

[13] D. Lifka, "The ANL/IBM SP scheduling system". In 1st Job Scheduling Strategies for Parallel Processing, pp. 295-303, Springer-Verlag, Apr 1995. LNCS 949.

[14] A. W. Mu'alem and D. G. Feitelson, "Utilization, predictability, workloads, and user runtime estimates in scheduling the IBM SP2 with backfilling". IEEE Trans. Parallel \& Distributed Syst. 12(6), pp. 529-543, Jun 2001 .

[15] "Parallel Workloads Archive". URL http://www.cs.huji.ac.il/labs/parallel/workload/.

[16] G. Sabin and P. Sadayappan, "On enhancing the reliability of job schedulers". In High Availability \& Performace Computing Workshop, Oct 2005.

[17] E. Shmueli and D. G. Feitelson, "Using site-level modeling to evaluate the performance of parallel system schedulers". In Modeling, Anal. \& Simulation of Comput. \& Telecomm. Syst., Sep 2006.

[18] S. Srinivasan, R. Kettimuthu, V. Subrarnani, and P. Sadayappan, "Characterization of backfilling strategies for parallel job scheduling". In Int'l Conf. Parallel Processing, pp. 514-522, Aug 2002.

[19] V. Subramani, R. Kettimuthu, S. Srinivasan, and P. Sadayappan, "Distributed job scheduling on computational grids using multiple simultaneous requests". In 11th IEEE Int'l Symp. High Performance Distributed Comput., p. 359, Jul 2002.

[20] T. Suzuoka, J. Subhlok, and T. Gross, Evaluating Job Scheduling Techniques for Highly Parallel Computers. Technical Report CMU-CS-95-149, School of Computer Science, Carnegie Mellon University, Aug 1995.

[21] D. Tsafrir, Y. Etsion, , and D. G. Feitelson, "A model/utility for generating user runtime estimates and appending them to a standard workload format (SWF) file". URL http://www.cs.huji.ac.il/labs/parallel/workload/m_tsafrir05, Feb 2006.

[22] D. Tsafrir, Y. Etsion, and D. G. Feitelson, "Backfilling using system-generated predictions rather than user runtime estimates". IEEE Trans. on Parallel \& Distributed Syst., 2007. To apper.

[23] D. Tsafrir, Y. Etsion, and D. G. Feitelson, "Modeling user runtime estimates". In 11th Job Scheduling Strategies for Parallel Processing, pp. 1-35, Springer-Verlag, Jun 2005. LNCS 3834.

[24] D. Tsafrir and D. G. Feitelson, "Instability in parallel job scheduling simulation: the role of workload flurries". In 20th IEEE Int'l Parallel \& Distributed Processing Symp., p. 10, Apr 2006.

[25] S. Vasupongayya, S-H. Chiang, and B. Massey, "Search-based job scheduling for parallel computer workloads". In IEEE Int'l Conf. Cluster Computing, Sep 2005.

[26] Y. Zhang, H. Franke, J. Moreira, and A. Sivasubramaniam, "Improving parallel job scheduling by combining gang scheduling and backfilling techniques". In 14th IEEE Int'l Parallel \& Distributed Processing Symp., pp. 133-142, May 2000.

[27] Y. Zhang, H. Franke, J. Moreira, and A. Sivasubramaniam, "An integrated approach to parallel scheduling using gang-scheduling, backfilling, and migration". IEEE Trans. Parallel \& Distributed Syst. 14(3), pp. 236-247, Mar 2003.

[28] D. Zotkin and P. J. Keleher, "Job-length estimation and performance in backfilling schedulers". In 8th IEEE Int'l Symp. High Performance Distributed Computing, p. 39, Aug 1999. 